\title{
AGRUPACIÓN FUNCIONAL DE ESPECIES VEGETALES PARA LA RESTAURACIÓN ECOLÓGICA DE ECOSISTEMAS DE MONTAÑA, BOGOTÁ, COLOMBIA
}

\author{
Functional grouping of plant species for the ecological restoration \\ of mountain ecosystems, Bogotá, Colombia
}

\author{
Maribel Vásquez-Valderrama' \& Jairo Solorza-Bejarano²
}

Vásquez-Valderrama, M., \& Solorza-Bejarano, J. (2018). Agrupación funcional de especies vegetales para la restauración ecológica de ecosistemas de montaña, Bogotá, Colombia. Colombia Forestal, 21(1), 5-17

Recepción: 3 de abril de 2017

\section{Resumen}

Los ecosistemas de montaña están sometidos a diversos factores de perturbación que requieren el desarroIlo de procesos de restauración ecológica, orientados a su recuperación estructural y funcional. En este estudio se analizaron los atributos funcionales de las especies vegetales de ocho especies vegetales incorporadas en áreas piloto de investigación en restauración ecológica presentes en Bogotá. Se registró el área foliar (AF), área foliar específica (AFE), contenido foliar de materia seca (CFMS), densidad de madera $(\mathrm{dB})$, altura máxima (Hmax) y hábito de crecimiento de Ageratina aristei, Abatia parviflora, Baccharis latifolia, Myrcianthes leucoxyla, Solanum oblongifolium, Vallea stipularis, Viburnum triphyllum y Xylosma spiculifera. Se encontraron tres grupos funcionales, los cuales presentan características de especies exclusivamente adquisitivas o adquisitiva-conservativa, lo que sugiere diferentes mecanismo y estrategias y mecanismos de adaptación a las condiciones de recuperación de las áreas perturbadas.

Palabras clave: altura máxima, área foliar específica, atributo funcional, contenido foliar, densidad de madera.
Aprobación: 28 de agosto de 2017

\begin{abstract}
Mountain ecosystems are subject to various factors of disturbance, therefore different ecological restoration processes should be developed for achieving their structural and functional recovery. In this study, we analyzed the functional attributes of eight plant species used in ecological restoration research areas in Bogotá. Leaf area (AF), leaf area of dry matter (CFMS), wood density $(\mathrm{dB})$, maximum height (Hmax) and growth habit of Ageratina aristei, Abatia parviflora, Baccharis latifolia, Myrcianthes leucoxyla, Solanum oblongifolium, Vallea stipularis, Viburnum triphyllum and Xylosma spiculifera were quantified. Three plant functional groups were found with exclusive characteristics of acquisitive or acquisitive-conservative species, suggesting different mechanisms and strategies of adaptation for the recovery of disturbed areas.
\end{abstract}

Key words: maximum height, functional attribute, specific foliar area, leaf content, wood density.

\footnotetext{
Jardín Botánico de Bogotá José Celestino Mutis. Bogotá, Colombia. mvasquez@jbb.gov.co Autor para correspondencia. Jardín Botánico de Bogotá José Celestino Mutis. Bogotá, Colombia. jsolorza@jbb.gov.co
} 


\section{INTRODUCCIÓN}

La biodiversidad hace referencia a elementos de la naturaleza que pueden ser descritos, clasificados, medidos y que están relacionados con procesos y funciones de los ecosistemas (Mayer, 2006). Representa un reto determinar la dinámica de la biodiversidad, los procesos del ecosistema y los factores abióticos (Loreau et al., 2001). La diversidad funcional combina diferentes tipos de procesos importantes en la estructura y estabilidad dinámica de una comunidad (Moore, 2001). Los efectos en el funcionamiento de los ecosistemas, relacionados con la abundancia, la composición, la distribución y las características de las especies, se pueden representar a través de atributos funcionales (Martín-López et al., 2007), compuestos de rasgos morfológicos y fisiológicos de los individuos y expresados en el crecimiento, la reproducción y la supervivencia (Violle et al., 2007; Duffy et al., 2007). Los atributos funcionales son, entonces, un indicador funcional de los ecosistemas dadas las características esenciales de las especies que permiten el equilibrio entre la adquisición y procesamiento de recursos (Leps et al., 2006).

Los rasgos más comunes en estudios sobre grupos funcionales corresponden a atributos de las hojas como el área foliar, el área foliar específica y el contenido foliar de materia seca (Wright et al., 2004). En la actualidad, se han incluido otros rasgos como la densidad de madera, la cual tiene una estrecha relación con la supervivencia y crecimiento de las plantas (Chave et al., 2009). Algunos rasgos hidráulicos del tallo como densidad y diámetro de vasos tienen implicaciones en la regulación hídrica de los individuos vegetales (Reich, 2014), ayudando a comprender con más detalle las relaciones de compromisos de las plantas (Díaz et al., 2016).

Es común encontrar agrupaciones de las especies presentes en los ecosistemas a partir de grupos funcionales, los cuales se pueden interpretar como un conjunto de especies que cumplen un rol funcional en un espacio definido (Hawkins et al.,
1989). De esta forma, el análisis de estos atributos se pueden considerar una herramienta efectiva para analizar la diversidad de respuestas eco-fisiológicas en los ecosistemas naturales (Chapin, 1993; Squeo et al., 1999) y en los procesos de restauración ecológica, este último, centrado en mejorar las relaciones de biodiversidad y los servicios de los ecosistemas (Benayas et al., 2009).

En los procesos de restauración ecológica, los atributos de los grupos funcionales se emplean como indicadores del funcionamiento del ecosistema y de los cambios generados por perturbaciones (Wortley et al., 2013; Murcia \& Guariguata, 2014; Ostertag et al., 2015), así como facilitadores y orientadores para la selección de especies (Barrera-Cataño \& Valdés-López, 2007; Castellanos-Castro \& Bonilla, 2011). El objetivo de este estudio fue caracterizar los atributos funcionales de las especies que con mayor frecuencia y abundancia se emplean en los procesos de restauración ecológica desarrollados por el Jardín Botánico de Bogotá José Celestino Mutis y sus implicaciones en la recuperación de ecosistemas de alta montaña.

\section{MATERIALES Y MÉTODOS}

\section{Área de estudio}

El Jardín Botánico de Bogotá cuenta con ocho áreas piloto de investigación en restauración ecológica (Apire) en del distrito capital y desde el año 2012 ha adelantado procesos en escenarios con diferentes factores de perturbación (v.g. cultivos agrícolas, ganadería, invasiones biológicas, plantaciones forestales exóticas e incendios). Las Apire (figura 1) se encuentran distribuidas en las localidades de Santa Fe (Parque Nacional Enrique Olaya Herrera y Venado de Oro), Suba (Las Mercedes), Engativá (La Florida), San Cristóbal (La Arboleda), Usme (Cantarrana y Arrayanes-Curubital) y Ciudad Bolívar (Mochuelo-Pasquilla-Mercedes) (Solorza, 2016). Corresponden a ecosistemas zonales 
de bosque andino y altoandino, entre los 2550 y 3200 m de altitud, con un comportamiento climático bimodal, de precipitación promedio anual de $1000 \mathrm{~mm}$, temperatura media anual entre los 12 y $15^{\circ} \mathrm{C}$, valores máximos de $25^{\circ} \mathrm{C}$ y mínimos por debajo de los $0^{\circ} \mathrm{C}$ (Barrera-Cataño et al., 2010).

\section{Registro y medición de rasgos funcionales}

En el periodo entre los años 2012 y 2015, el Jardín Botánico de Bogotá ha desarrollado la incorporación de 119689 individuos vegetales, correspondientes a 113 especies y 49 familias, con el objetivo de recuperar áreas con diferentes factores de perturbación. Las especies de estudio fueron seleccionadas de acuerdo con la frecuencia relativa de uso en al menos siete de las ocho Apire, donde el registro de muestras se realizó con individuos juveniles de las especies seleccionadas.
A las especies seleccionadas se les registró atributos funcionales de fácil medición (Casanoves et al., 2011). Se trabajó con seis atributos en total, tres foliares, uno de tallo y dos de historia de vida. Para los rasgos foliares se seleccionaron cinco individuos por especie y cinco hojas por individuo, a los que se les midió el área foliar especifica (AFE), el área foliar (AF) y el contenido foliar de materia seca (CFMS) mediante el empleo del software libre ImageJ (Schneider, 2012), y determinados a partir de los protocolos sugeridos por Corneliessen et al. (2003) y Salgado-Negret (2015).

Los rasgos de tallo y de historia de vida tales como densidad de madera $(\mathrm{dB})$, altura máxima (Hmax) y hábito de crecimiento, se tomaron de registros publicados para las especies. En los casos en los que no se encontró información específica de la especie se trabajó con información del género. Se utilizó el sistema APG III para la clasificación

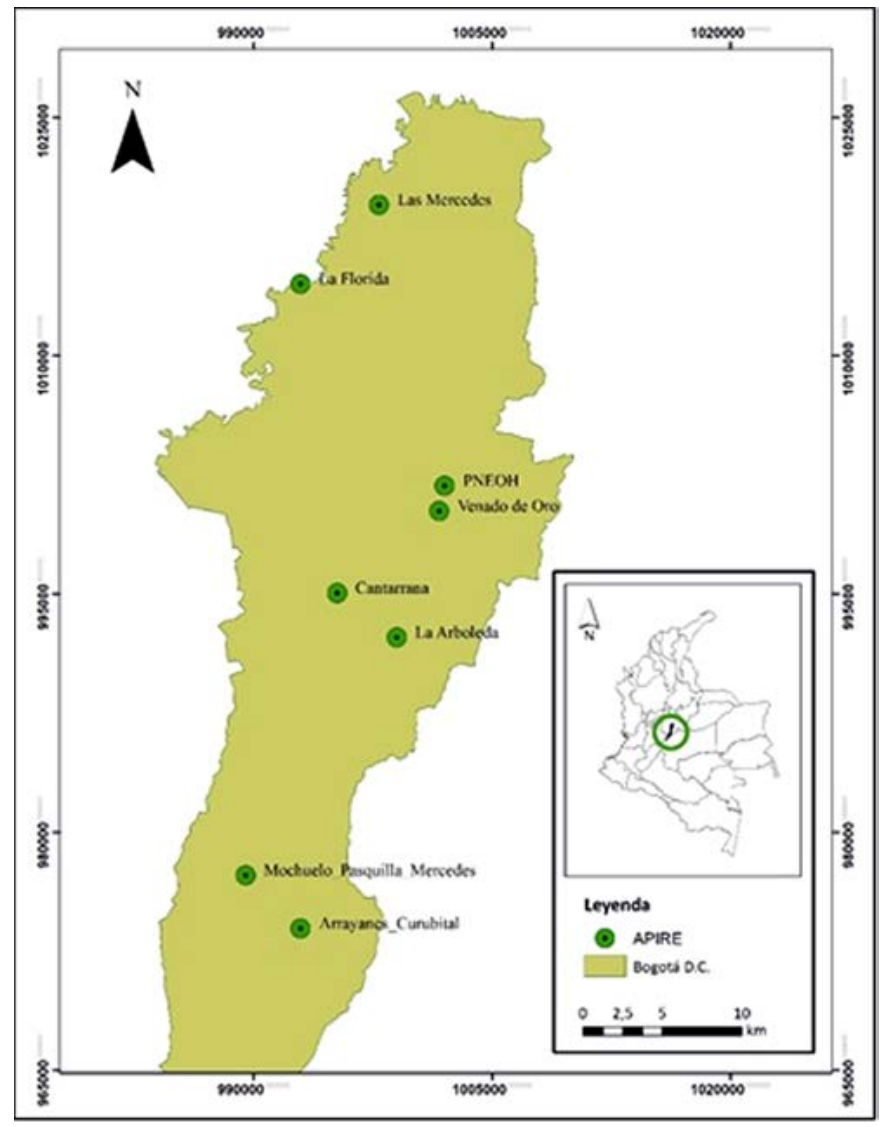

Figura 1. Áreas piloto de investigación en restauración ecológica en Bogotá, Colombia. 
de especies (The Angiosperm Phylogeny Group, 2009), donde el clúster indica las relaciones de parentesco filogenético de las especies en estudio, construido a partir de las entidades taxonómicas de las especies y del árbol filogenético dispuesto por el software Phylocom (Webb et al., 2008).

\section{Análisis de datos}

Se realizaron análisis de varianza para determinar diferencias de los rasgos funcionales entre las especies y dentro de la especie. Cuando no se cumplieron los supuestos estadísticos se realizaron transformaciones log10 para el análisis. Se emplearon pruebas postHoct para determinar diferencias entre pares de especies (mínima diferencia significativa de Fisher-LSD), y prueba Honesta de Tukey HDS. Se construyeron análisis de componentes principales (ACP) para identificar agrupaciones de las especies respecto a sus atributos funcionales. Finalmente, se realizaron clúster de las especies de acuerdo con sus abundancias y presencia en las áreas, identificando relaciones de parentesco (filogenético) y grupos funcionales. Todos los análisis se corrieron en el software estadístico R Project $@$ (R Core Team, 2016).

\section{RESULTADOS}

De las 11 especies que representan el $28.4 \%$ de los individuos sembrados en al menos siete Apire, entre el año 2012 y 2015, se seleccionaron ocho para caracterizar sus rasgos funcionales: $A$. aristei (2120 ind), A. parviflora (1763 ind), B. latifolia (2740 ind), M. leucoxyla (2838 ind), S.oblongifolium (11 073 ind), V. stipularis (2134 ind), V. triphyllum (3456 ind) y X. spiculifera (2565 ind), excluyendo a Smallanthus pyramidalis (2 672 ind), Verbesina crassiramea (1858) y Clusia multiflora (807 ind), ya que no se contó con individuos juveniles para el registro de información (figura 2).

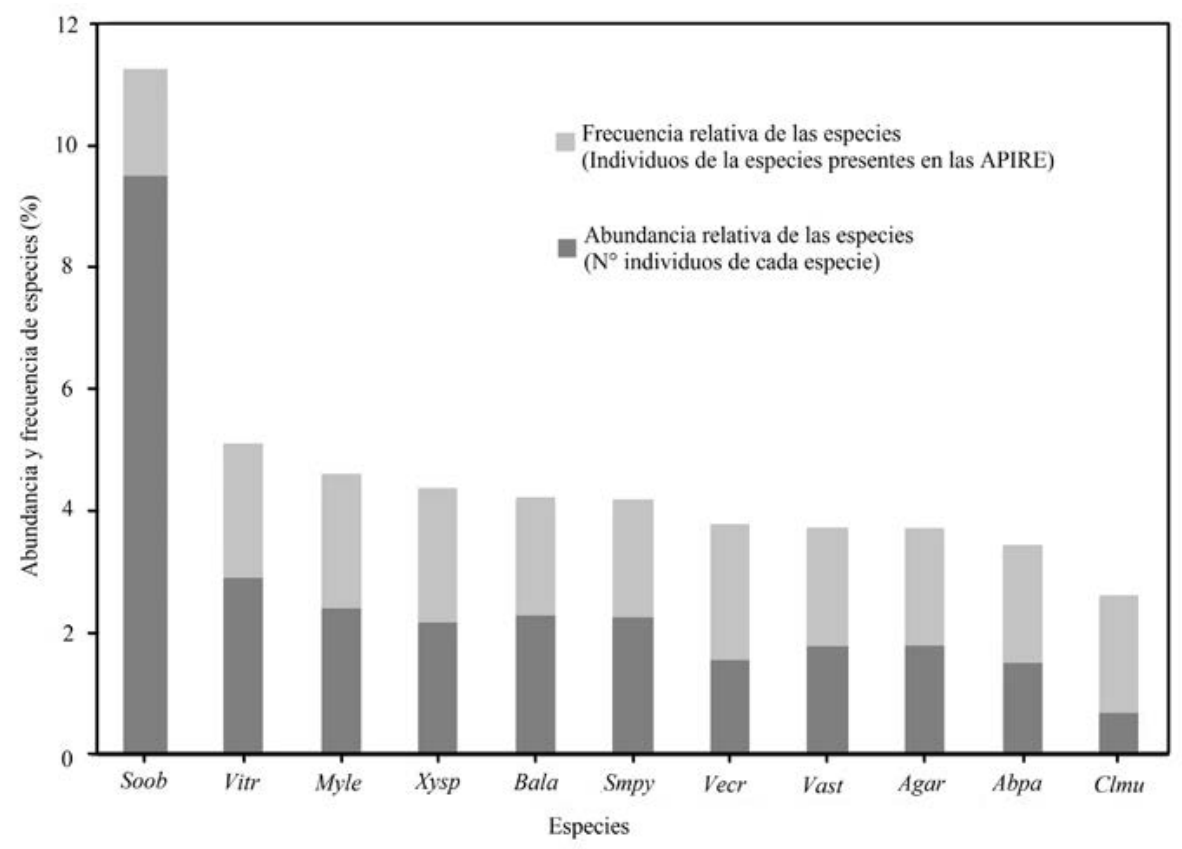

Figura 2. Porcentaje de abundancia de las especies en los procesos de restauración realizados por el Jardín Botánico de Bogotá entre el año 2012 y 2015, Agar (A. aristei), Abpa (A. parviflora), Bala (B. latifolia), Clmu (C. multiflora), Myle (M. leucoxyla), Smpy (S. pyramidalis), Soob (S.oblongifolium), Vast (V. stipularis), Vecr (V. crassiramea), Vitr (V. triphyllum) y Xysp (X. spiculifera). 
El $71.6 \%$ restante corresponde a 102 especies (85 665 ind), cada una de estas aporta menos del $0.06 \%$ de los individuos reintroducidos.

Los individuos de las especies $M$. leucoxyla y $X$. spiculifera presentan áreas foliares más pequeñas $\left(0.59 \pm 0.11\right.$ y $0.72 \pm 0.09 \mathrm{~mm}^{2}$, respectivamente $)$ y se diferenciaron significativamente del resto de las especies $(\mathrm{P}<0.001)$. Las otras especies presentaron áreas foliares entre $0.73 \pm 0.18$ y $1.74 \pm 0.53$ $\mathrm{mm}^{2}$, diferenciándose tan solo en pares particulares. Sin embargo, S. oblongifolium con $1.31 \pm 0.33$ $\mathrm{mm}^{2}$ registró el mayor valor para este atributo. En contraste, para AFE solo B. latifolia (238.02 \pm 43.85 $\left.\mathrm{cm}^{2} \cdot \mathrm{g}^{-1}\right)$ y X. spiculifera $\left(106.43 \pm 20.33 \mathrm{~cm}^{2} \cdot \mathrm{g}^{-1}\right)$ son significativamente diferentes de las demás especies $(\mathrm{P}<0.001)$. El comportamiento del CFMS fue similar al AFE para todas las especies (figura 3).

V. stipularis presenta el mayor $\mathrm{dB}\left(0.62 \mathrm{~g} . \mathrm{cm}^{-3}\right)$ y $S$. oblongifolium el menor $\mathrm{dB}\left(0.41 \mathrm{~g} . \mathrm{cm}^{-3}\right)$, aspecto que puede estar relacionado con las estrategias de adaptación de las especies; en general, las especies presentan densidades entre 0.45 y 0.58 g.cm ${ }^{-3}$. En altura, las especies M. leucoxyla y $A$. parviflora son las especies que alcanzan el estrato
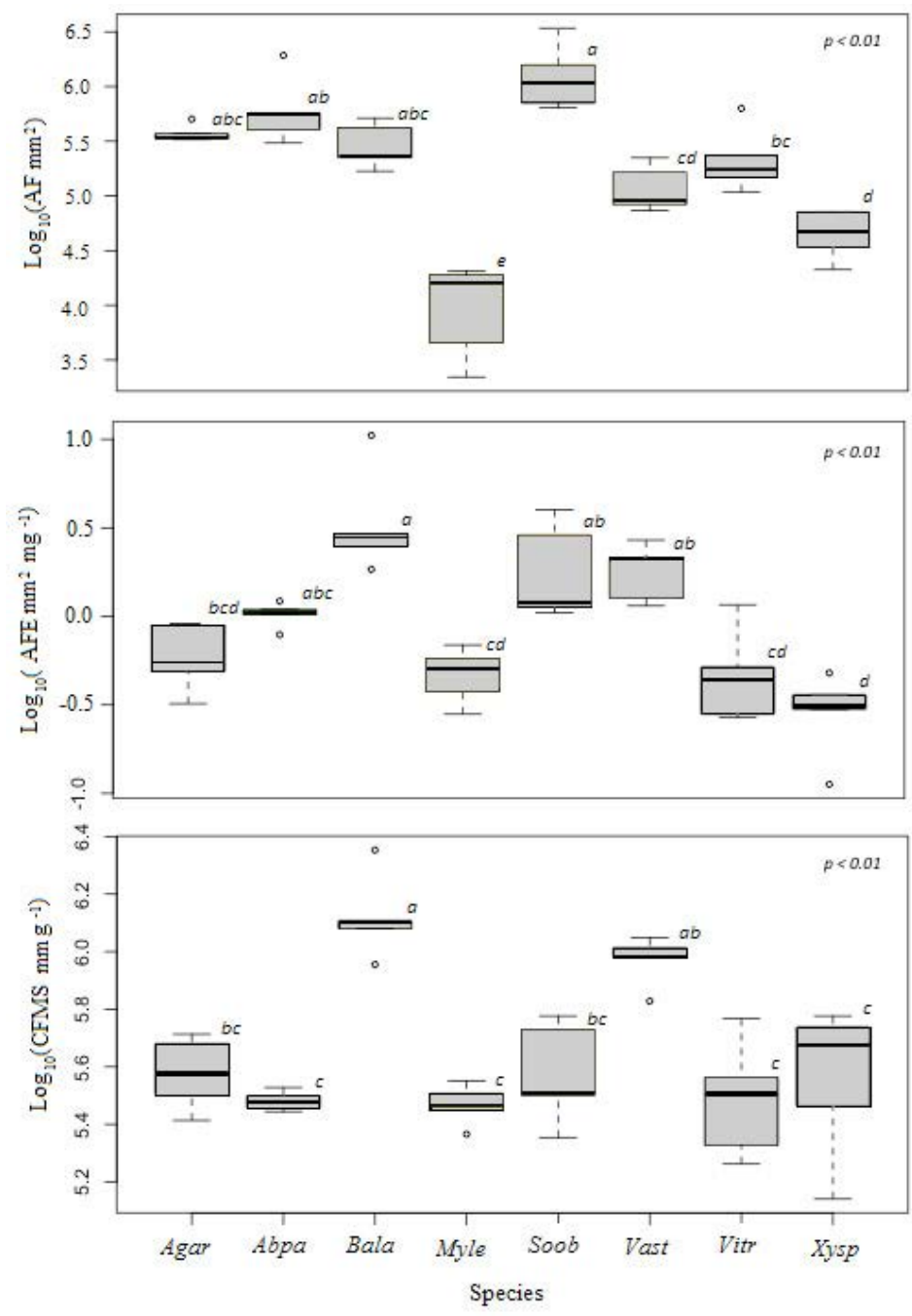

Figura 3. Atributos foliares por especie, AF (área foliar), AFE (área foliar específica), CFMS (contenido foliar de materia seca, Agar (A. aristei), Abpa (A. parviflora), Bala (B. latifolia), Myle (M. leucoxyla), Soob (S. oblongifolium), Vast (V. stipularis), Vitr (V. triphyllum) y Xysp (X.spiculifera), especies con letras diferentes presentan diferencias significativas. 
arbóreo (25 y 20 m respectivamente), mientras que $A$. aristei y $B$. latifolia son especies arbustivas que alcanzan 3.5 y $4 \mathrm{~m}$ (figura 4 ).

En el ACP, que explica el $76 \%$ del comportamiento de las especies $(32 \%$ en el componente uno y del $44 \%$ en el componente dos), se lograron identificar tres grupos principales de plantas: el primer grupo está conformado por las especies B. latifolia y V. stipularis, las cuales están relacionadas con altos valores de CFMS y AFE; el segundo grupo se conforma por M. leucoxyla, A. aristei, $V$. triphyllum y $X$. spiculifera, relacionados con valores de altura y altos valores de $\mathrm{dB}$; y un tercer grupo conformado por S. oblongifolium y A. parviflora, con los mayores valores de AF (figura 5).

De acuerdo al análisis del clúster, en el primer conglomerado se muestra la agrupación de las especies de acuerdo a la abundancia de cada una de las zonas, donde se generan tres grupos principales. El primero de especies abundantes con más del $26 \%$ del total de individuos (V. triphyllum, $M$. leucoxyla y X. spiculifera); el segundo, de la especie más abundante con el $32.5 \%$ del total de individuos y presente en las ocho Apire (S. oblongifolium); y el tercero, especies con abundancia entre el $5 \%$ y $6 \%$ del total de individuos, y presentes
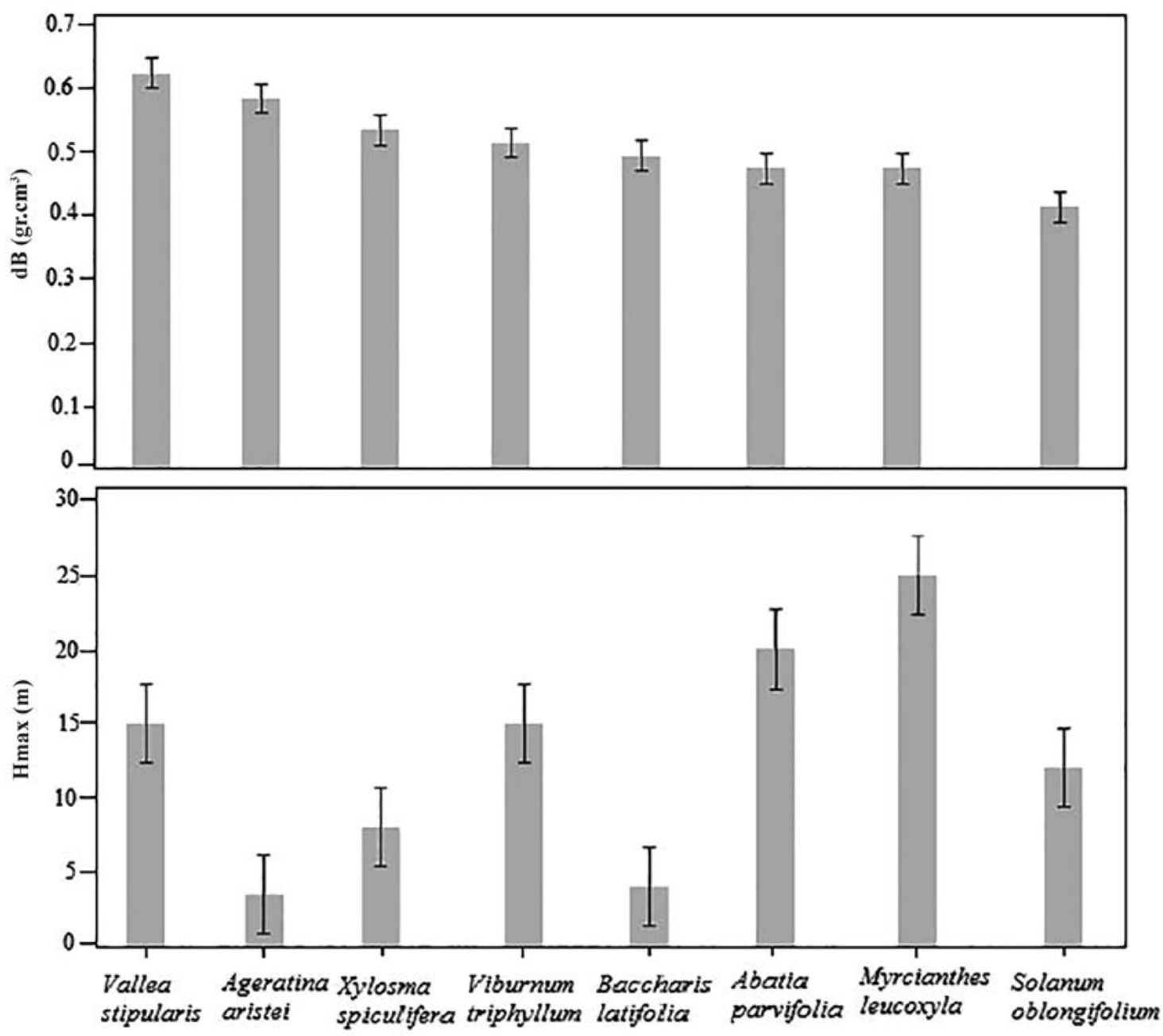

Figura 4. Densidad básica y altura por especie, dB (densidad básica de la madera), Hmax (altura máxima que puede alcanzar los individuos de la especie). 
en al menos siete de las ocho áreas (B. latifolia, $A$. parviflora, A. aristei y V. stipularis) (figura 5).

En el segundo conglomerado se puede apreciar parentesco de las especies (divergencia filogenética). El clúster indica menor relación entre especies en las medidas que hay mayor separación de las ramas, donde se conforman tan solo dos grupos, lo que demuestra que, aunque se empleen diferentes especies a nivel filogenético la diversidad trabajada en los procesos de restauración ecológica es baja. En contraste en el último conglomerado, se encuentra la agrupación de las especies a partir de las características funcionales, identificando los tres grupos encontrados en la misma agrupación que en ACP. Lo que nos muestra que, aunque la diversidad filogenética es baja, a nivel funcional se presentan tres grupos definidos que varían en sus características y, por tanto, existe una compensación a nivel funcional de las especies (figura 6).

Pese a que la riqueza de especies es alta (113 especies) la abundancia de individuos está concentrada en pocas especies (11 especies), lo que nos muestra que la diversidad funcional y filogenética es baja, pero teniendo en cuenta los objetivos a corto plazo de los procesos de restauración ecológica que buscan recuperar la cobertura vegetal, estructura del suelo y control de factores de tensión, fundamental en las primeras etapas en los que se encuentran los procesos de restauración ecológica con menos de cuatro años de implementación.

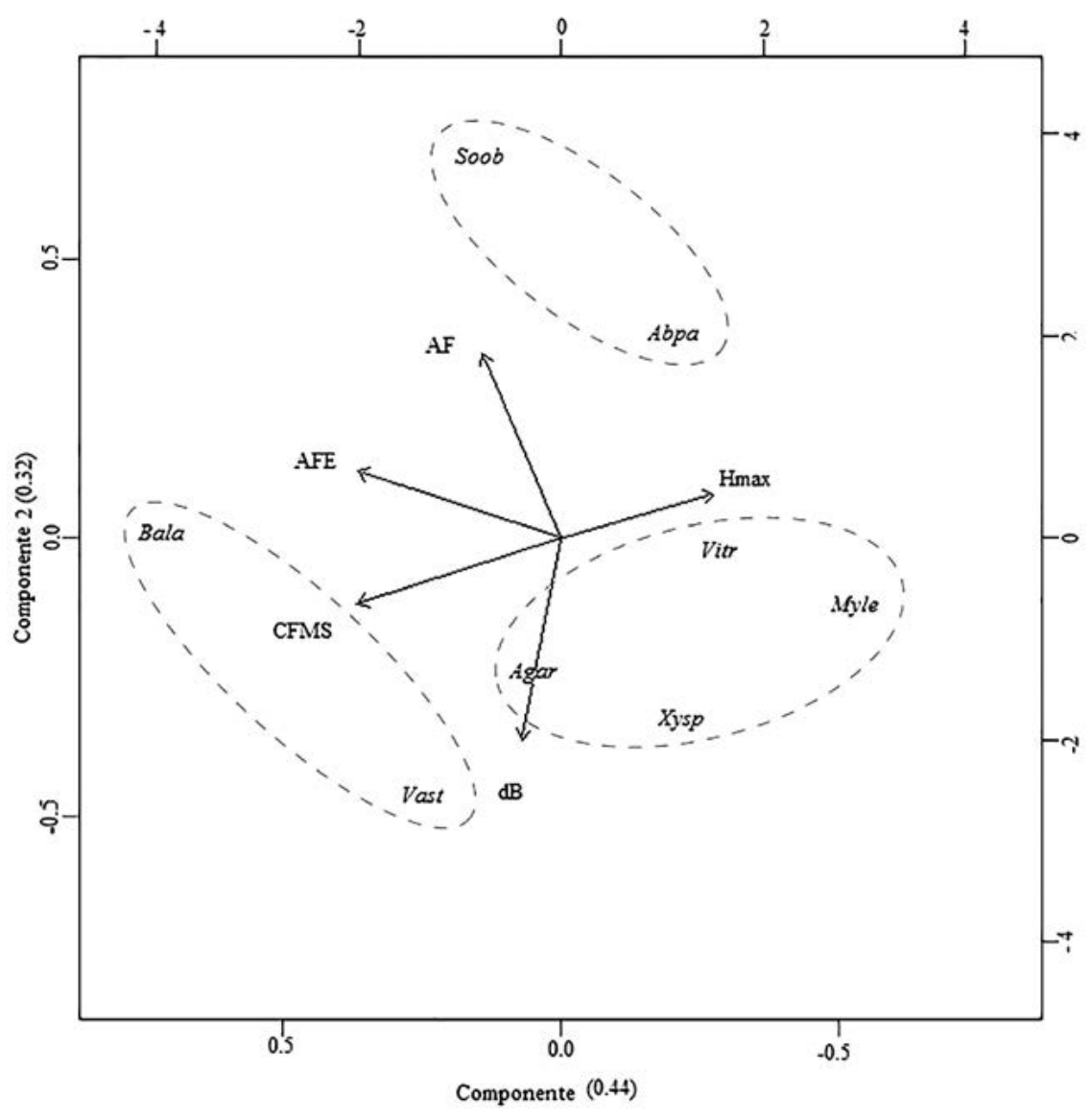

Figura 5. Análisis de componentes principales, Agar (A. aristei), Abpa (A. parviflora), Bala (B. latifolia), Myle (M. leucoxyla), Soob (S. oblongifolium), Vast (V. stipularis), Vitr (V. triphyllum) y Xysp (X. spiculifera). dB (densidad básica de la madera), AF (área foliar), AFE (Área foliar especifica), CFMS (contenido foliar de materia seca), Hmax (altura máxima de las especies). 

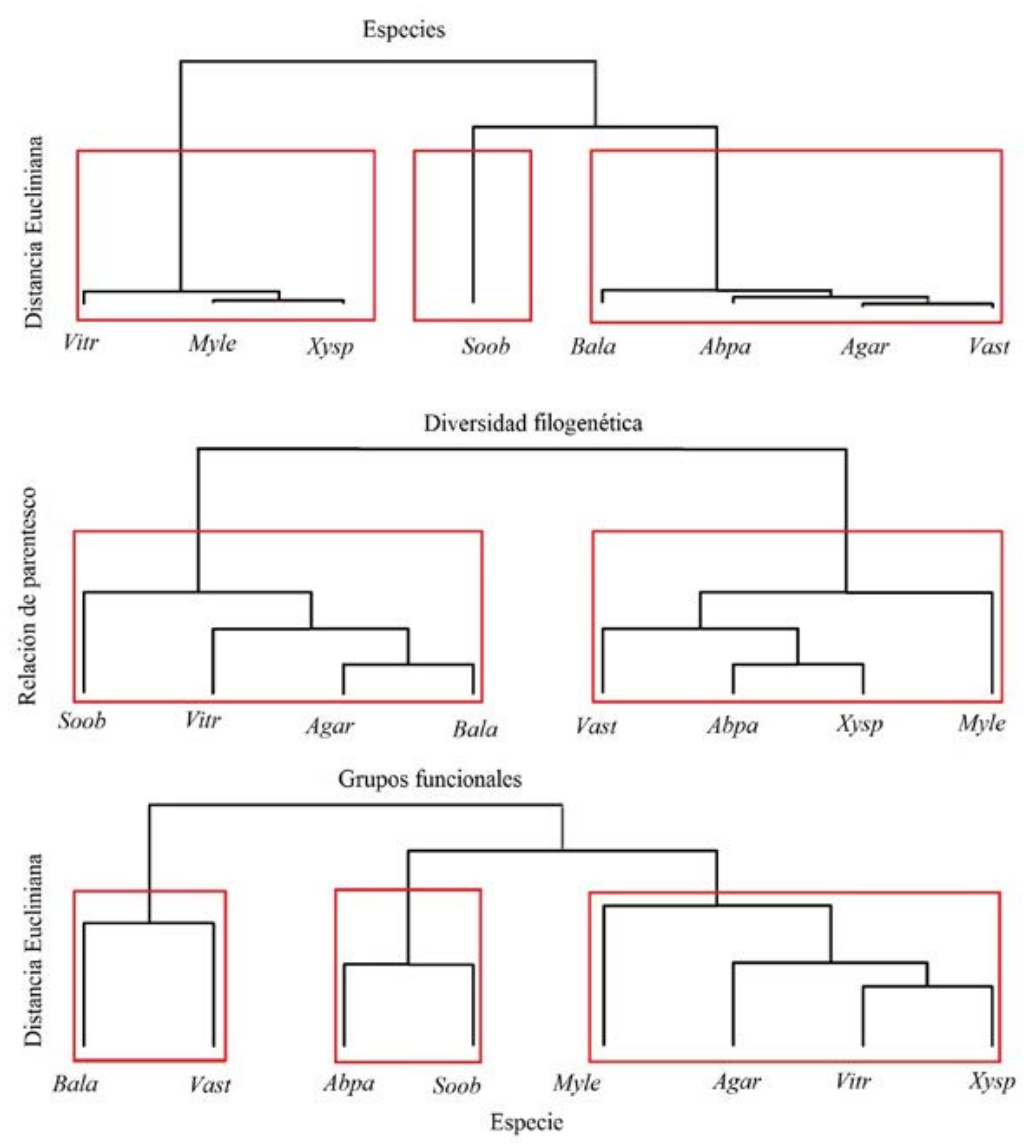

Figura 6. Análisis de conglomerado de acuerdo a la frecuencia y abundancia de las especies en las APIRE, diversidad filogenética y atributos funcionales, Agar (A. aristei), Abpa (A. parviflora), Bala (B. latifolia), Myle (M. leucoxyla), Soob (S. oblongifolium), Vast (V. stipularis), Vitr (V. triphyllum) y Xysp (X. spiculifera).

\section{DISCUSIÓN}

Las ocho especies más empleadas en las áreas de restauración ecológica que el Jardín Botánico de Bogotá ha reintroducido entre los años 2012 a 2015, conforman tres grupos que presentan diferencias a nivel funcional dentro de las áreas, generando limitaciones y oportunidades para su establecimiento.

El primer grupo lo conforman las especies $S$. oblongifolium y $A$. parviflora que presentan los mayores valores de AF, las cuales son más resistentes a áreas frías, presentan mayor capacidad fotosintética indispensable para la creación de tejidos y aspectos productivos de las plantas, con mayor captación y uso de los recursos, manteniendo altas tasas de adquisición de recursos, equilibrando los costos de construcción de la hoja con el potencial de crecimiento. (Wilson et al., 1999; Lüscher et al., 2001; Warnock et al., 2006; Díaz et al., 2016). Adicionalmente, se encontró que las especies que presentan mayor tamaño de hoja (AF), presentan menor densidad de madera, respuesta que se debe a la inversión de más energía en mantener altas tasas de recambio, hojas poco costosas y altas tasas fotosintéticas para maximizar la captura de recursos, más que en generar tallos con altas resistencias comportándose como especies más adquisitivas (Pickup et al., 2005; Lohbeck et al., 2015).

El segundo grupo conformado por B. latifolia y $V$. stipularis relaciona las especies con mayores AFE, asociadas a mejor uso de recursos, produciendo 
más hojas, pero siendo más susceptibles a la herbívora y con menor longevidad foliar, por lo que son recomendadas para ambientes con muchos nutrientes. En contraste las especies con menor AFE (V. triphyllum, X. spiculifera, A. aristei y M. leucoxyla) se concentran en la retención de recursos, siendo más apropiadas en zonas con menor disponibilidad de nutrimentos (Poorter \& De Jong, 1999; Wilson et al., 1999; Marenco et al., 2009). El AFE se relaciona de manera directa con la disponibilidad de recursos y con la disponibilidad de lumínica (Hodgson et al., 2011), además de relacionarse de manera positiva con los contenidos de nitrógeno en las hojas (Gulías et al., 2003). sin embargo, no se relaciona con el tamaño de hoja (Wilson et al., 1999; Ackerly et al., 2002). En el trabajo desarrollado por Melo-Cruz et al. (2012), para cinco especies nativas andinas de la cordillera oriental en áreas en proceso de restauración ecológica, se registró variabilidad en las características foliares, aspecto similar al encontrado para las especies evaluadas en este estudio, donde se incluyen especies de diferente origen taxonómico y características funcionales.

El tercer grupo está conformado por $M$. leucoxyla, A. aristei, V. triphyllum y X. spiculifera, y se relacionan con mayores valores de Hmax y dB. Las especies tolerantes a la luz o pioneras, presentan una relación inversa con la dB (Gelder et al., 2006), las especies que requieren luz (King et al., 2005) y tiene poca competencia intra e interespecífica (Kunstler et al., 2015), presentan un mayor crecimiento en altura y una alta capacidad fotosintética, y se caracterizan por tener bajas. Por otro lado, especies con altas $\mathrm{dB}$ se ha observado que tiene una relación con la tolerancia a la sequía (Hacke et al., 2001). En general, el grupo de especies en nuestro estudio que se relaciona con la densidad de madera presenta densidades entre 0.45 y 0.60 g.cm ${ }^{-3}$, densidad considerada como media, lo que indica que presentan compensaciones entre características adquisitivas y características conservativas que les permiten adaptarse al medio (Puertas et al., 2013).
A nivel filogenético se encuentran dos grupos y se ha reportado que especies con relaciones estrechas en su filogenia tienden también a presentar características similares en sus rasgos funcionales (Ackerly, 2009; Flynn et al., 2011). No obstante, como se aprecia en este estudio, estas similitudes no se observaron ya que existen diferencias a nivel de grupo funcional. Es posible que se deba a las adaptaciones de las especies en respuesta a factores ambientales, debido a que existen múltiples combinaciones de los atributos funcionales para cumplir una misma función o proceso determinado (Díaz et al., 2013). Ningún atributo aislado puede cumplir con procesos o funciones ecosistémicas, por el contrario, requiere la combinación y compensaciones de diferentes estrategias y rasgos para cumplir con ello. Así que los tres grupos funcionales encontrados y su filogenia, muestra agrupaciones de especies que están emparentadas y cumplen funciones similares, pero que pueden emplear estrategias diferentes (variación de rasgos funciones entre las especies), para cumplir su función en el ecosistema (Maherali \& Klironomos, 2007).

\section{CONCLUSIONES}

Se identificaron tres grupos funcionales. El primero de ellos está relacionado con el AF, se trata de especies tolerantes a la luz, con rápido crecimiento y tasas de recambio altas comportándose como especies adquisitivas. Un segundo grupo relacionado a AFE y CFMS, con especies de altos contenidos nutricionales en las hojas y menor longevidad foliar; y un tercer grupo relacionado a la Hmax de las especies y la $d B$, donde las especies con menores densidades presentan mayores crecimientos y menor altura. Los dos últimos grupos presentan características de especies adquisitivas y conservativas.

La respuesta de las especies que presentan ya sea de características adquisitivas o conservativas, se constituye en un factor importante a tener en cuenta para la selección de especies y su incorporación en diferentes espacios y momentos del 
proceso de restauración ecológica. Además, se debe tener en cuenta el grado de perturbación del área, la disponibilidad de recursos en el medio, la estructura y composición de la cobertura vegetal establecida y la funcionalidad del sistema en su conjunto.

Adicionalmente, se encontró que, aunque las especies empleadas en los procesos de restauración ecológica conforman dos grupos de acuerdo a sus similitudes filogenéticas: a nivel funcional aumenta el número en un grupo, indicando que las especies presentan diferentes mecanismos y estrategias de adaptación, lo que corrobora que la combinación de diferentes atributos funcionales y en diferentes proporciones pueden contribuir a diferentes procesos ecosistémicos. Aunque el mayor número de individuos reintroducidos en las Apire involucra pocas especies, se aporta a la recuperación de las funciones ecológicas gracias a la combinación de atributos funcionales de estas especies. La estrategia de las especies empleadas está asociada a la adquisición de recursos, estrategia indispensable para la recuperación de zonas disturbadas ya que induce una rápida recuperación de la cobertura vegetal. Sin embargo, es claro que la restauración es a largo plazo, por lo cual se recomienda involucrar grupos funcionales con rasgos conservativos, de tal manera que se continúe con los procesos de restauración ecológica, teniendo en cuenta el sistema de referencia, los mecanismos ecológicos que se deseen facilitar de acuerdo con los objetivos planteados para cada escenario.

Para activar el proceso sucesional en la fase inicial de implementación de las actividades de restauración ecológica, se incorporó un grupo de especies dominantes con la finalidad de actuar como especies facilitadoras en la recuperación de la cobertura vegetal, estructura del suelo y control de los factores de tensión. Una vez concluida esta fase, y como parte de la evaluación, se recomienda continuar con la incorporación de otras especies e incrementar el número de individuos de las especies que se han incluido en las Apire, pero no se incluyen en este análisis, dado que aportan nuevos componentes y características estructurales y funcionales que dan mayor complejidad al ecosistema, permitiendo mayor autosostenibilidad.

\section{CONFLICTO DE INTERESES}

Los autores declaran no tener conflicto de intereses.

\section{CONTRIBUCIÓN POR AUTOR}

El autor único es responsable de la obra en todos los aspectos que condujeron a la elaboración de su publicación.

\section{REFERENCIAS BIBLIOGRÁFICAS}

\section{Ackerly, D., Knight, C., Weiss, S., Barton, K., \& Starmer,} K. (2002). Leaf size, specific leaf area and microhabitat distribution of chaparral woody plants: Contrasting patterns in species level and community level analyses. Oecologia, 130(3), 449-457. DOI: https://doi.org/10.1007/s004420100805

Ackerly, D. (2009). Conservatism and diversification of plant functional traits: Evolutionary rates versus phylogenetic signal. Proceedings of the National Academy of Sciences of the United States of America, 106,19699-19706. DOI: https://doi. org/10.1073/pnas.0901635106

Barrera-Cataño, J., \& Valdés-López, C. (2007). Herramientas para abordar la restauración ecológica de áreas disturbadas en Colombia. Universitas Scientiarum, 12(2), 11-24.

Barrera-Cataño, J., Contreras-Rodríguez, S., Garzón-Yepes, N., Moreno-Cárdenas, A., \& Montoya-Villarreal, S. (2010). Manual para la restauración ecológica de los ecosistemas disturbados del Distrito Capital. Bogotá: Secretaría Distrital de Ambiente-Pontificia Universidad Javeriana. 403 p.

Benayas, R., Newton, A., Diaz, A., \& Bullock, J. (2009). Enhancement of biodiversity and ecosystem 
services by ecological restoration: a meta-analysis. Science, 325(5944), 1121-1124. DOI: https://doi. org/10.1126/science.1172460

Castellanos-Castro, C., \& Bonilla, M. (2011). Grupos funcionales de plantas con potencial uso para la restauración en bordes de avance de un bosque altoandino. Acta Biológica Colombiana, 16(1), 153-174.

Casanoves, F., Pla, L., \& Rienzo, J. (eds.). (2011). Valoración y análisis de la diversidad funcional y su relación con los servicios ecosistémicos. Turrialba, Costa Rica: Centro Agronómico Tropical de Investigación y Enseñanza (Catie). 834 p.

Chapin F. (1993). Functional role of growth forms in ecosystem and global processes. In Scaling Physiological Processes: Leaf to Globe, 287-312. DOI: https:// doi.org/10.1016/B978-0-12-233440-5.50024-5

Chave, J., Coomes, D., Jansen, S., Lewis, S. L., Swenson, N., \& Zanne, A. (2009). Towards a worldwide wood economics spectrum. ECOlogy Letters, 12(4), 351-366. DOI: https://doi. org/10.1111/j.1461-0248.2009.01285.x

Corneliessen, J., Lavorel, S., Garnier, E., Díaz, S., Buchmann, N., Gurvich, D., Reich, P., Ter Steege, H., Morgan, H., Heijden, M., Pausas, J., \& Poorter, H. (2003). A handbook of protocols for standardised and easy measurement of plant functional traits worldwide. Australian Journal of Botany, 51, 335380. DOI: https://doi.org/10.1071/BT02124

Díaz, S., Kattge, J., Cornelissen, J., Wright, I., Lavorel, S., Dray, S., \& Garnier, E. (2016). The global spectrum of plant form and function. Nature, 529(7585), 167-171. DOI: https://doi.org/10.1038/ nature16489

Díaz, S., Purvis, A., Cornelissen, J., Mace, G., Donoghue, M., Ewers, R., \& Jordano, P. (2013). Functional traits, the phylogeny of function, and ecosystem service vulnerability. Ecology and Evolution, 3(9), 2958-2975. DOI: https://doi.org/10.1002/ece3.601

Duffy, J., France, K., McIntyre, P., Thebault, E., \& Loreau, M. (2007). The functional role of biodiversity in ecosystems: incorporating trophic complexity. Ecology Letters. 10, 522-538. DOI: https:// doi.org/10.1111/j.1461-0248.2007.01037.x
Flynn, D., Mirotchnick, N., Jain, M., Palmer, M., \& Naeem, S. (2011). Functional and phylogenetic diversity as predictors of biodiversity-ecosystem-function relationships. Ecology, 92(8), 1573-1581. DOI: https://doi.org/10.1890/10-1245.1

Gelder, H., Poorter, L., \& Sterck, F. (2006). Wood mechanics, allometry, and life-history variation in a tropical rain forest tree community. The New phytologist, 171(2), 367-78. DOI: https://doi. org/10.1111/j.1469-8137.2006.01757.x

Gulías, J., Flexas, J., Mus, M., Cifre, J., Lefi, E., \& Medrano, H. (2003). Relationship between maximum leaf photosynthesis, nitrogen content and specific leaf area in Balearic endemic and non-endemic Mediterranean species. Annals of Botany, 92(2), 215222. DOI: https://doi.org/10.1093/aob/mcg123

Hacke, U., Sperry, J., Pockman, W., Davis, S., \& Mc CuIloh, K. (2001). Trends in wood density and structure are linked to prevention of xylem implosion by negative pressure. Oecologia, 126(4), 457-461. DOI: https://doi.org/10.1007/s004420100628

Hawkins C., \& Macmahon, J. (1989). Guilds: the multiple meanings of a concept. Annual Review of Entomology, 34, 423-451. DOI: https://doi.org/10.1146/ annurev.en.34.010189.002231

Hodgson, J., Montserrat-M, G., Charles, M., Jones, G., Wilson, P., Shipley, B., \& Sharafi, M. (2011). Is leaf dry matter content a better predictor of soil fertility than specific leaf area? Annals of Botany, 108(7), 1337-1345. DOI: https://doi.org/10.1093/aob/ mer225

King, D., Davies, S., Nur Supardi, M., \& Tan, S. (2005). Tree growth is related to light interception and wood density in two mixed dipterocarp forests of Malaysia. Functional Ecology, 19(3), 445-453. DOI: https://doi.org/10.1111/j.1365-2435.2005.00982.x

Kunstler, G., Falster, D., Coomes, D., Hui, F., Kooyman, R., Laughlin, D., Poorter, L. (2015). Plant functional traits have globally consistent effects on competition. Nature, 529(7585), 1-15. DOI: https://doi. org/10.1038/nature16476

Leps, J., Bello, F., Lavorel, S., \& Berman, S. (2006). Quantifying and interpreting functional diversity of 
natural communities: practical considerations matter. Preslia, 78, 481-501.

Loreau, M., Naeem, S., Inchausti, P., Bengtsson, J., Grime, J., Hector, A., \& Hooper, D. (2001). Biodiversity and ecosystem functioning: current knowledge and future challenges. Science, 294(5543), 804-8. DOI: https://doi.org/10.1126/science.1064088

Lohbeck, M., Lebrija-Trejos, E., Martínez-Ramos, M., Meave, J., Poorter L., \& Bongers, F. (2015). Functional trait strategies of trees in dry and wet tropical forests are similar but differ in their consequences for succession. PloS one, 10(4), e0123741. DOI: https://doi.org/10.1371/journal.pone.0123741

Lüscher, A., Stäheli, B., Beaun, R., \& Nösberger, J. (2001). Leaf area, competition with grass, and clover cultivar: Key factors to successful overwintering and fast regrowth of white clover (Trifolium repens L.) in spring. Annals of Botany, 88, 725-735. DOI: https://doi.org/10.1006/anbo.2001.1509

Maherali, H., \& Klironomos, J. (2007). Influence of phylogeny on fungal community assembly and ecosystem functioning. Science, 316, 1746-1748. DOI: https://doi.org/10.1126/science.1143082

Marenco, R., Antezana-Vera, S., \& Nascimento, H. (2009). Relationship between specific leaf area, leaf thickness, leaf water content and SPAD-502 readings in six Amazonian tree species. Photosynthetica, 47(2), 184-190. DOI: https://doi.org/10.1007/ s11099-009-0031-6

Martín-López, B., González, J., Díaz, S., Castro, I., \& García-Llorente, M. (2007). Biodiversidad y bienestar humano: el papel de la diversidad funcional. Ecosistemas, 16(3), 69-80.

Mayer, P. (2006). Biodiversity-The appreciation of different thought styles and values helps to clarify the term. Restoration Ecology, 14(1), 105-111. DOI: https://doi.org/10.1111/j.1526-100X.2006.00111.x

Melo-Cruz, O., Rodríguez-Santos, N., \& Rojas-Ramírez, F. (2012). Patrones de arquitectura foliar asociados al crecimiento funcional de cinco especies leñosas nativas de la cordillera oriental utilizadas en restauración ecológica en la sabana de Bogotá. Colombia Forestal, 15(1), 119-130. DOI: https://doi.org/10.14483/udistrital.jour.colomb. for.2012.1.a04

Moore, J. (2001). Diversity taxonomic versus Functional. University of Northern Colorado. Encyclopedia of Biodiversity. vol II. Colorado, EE. UU. p 205-2015. DOI: https://doi.org/10.1016/ B0-12-226865-2/00078-X

Murcia, C., \& Guariguata, M. (2014). La restauración ecológica en Colombia: tendencias, necesidades y oportunidades. Occasional Paper. Bogor, Indonesia: Centro para la investigación Forestal Internacional (Cifor). $107 \mathrm{p}$.

Ostertag, R., Warman, L., Cordell, S., \& Vitousek, P. (2015). Using plant functional traits to restore Hawaiian rainforest. Journal of Applied Ecology, 52(4), 805-809. DOI: https://doi. org/10.1111/1365-2664.12413

Poorter, H., \& De Jong, R. (1999). A comparison of specific leaf area, chemical composition and leaf construction costs of field plants from 15 habitats differing in productivity. New Phytologist, 143(1), 163-176. DOI: https://doi. org/10.1046/j.1469-8137.1999.00428.x

Puertas, P., Guevara, C., \& Espinoza, M. (2013). Manual de transformación de maderas. Lima: Organización Internacional de Maderas (OIMT). 122 p.

Pickup, M., Westoby, M., \& Basden, A. (2005). Dry mass costs of deploying leaf area in relation to leaf size. Functional Ecology, 19(1), 88-97. DOI: https:// doi.org/10.1111/j.0269-8463.2005.00927.x

Reich, P. (2014). The world-wide 'fast-slow' plant economics spectrum: a traits manifesto. Journal of Ecology, 102, 275-301. DOI: https://doi. org/10.1111/1365-2745.12211

R Core Team. (2016). R: A language and environment for statistical computing. R Foundation for statistical Computing, Vienna, Austria. Recuperado de: https://www.R-project.org/

Salgado-Negret, B. (ed). (2015). La ecología funcional como aproximación al estudio, manejo y conservación de la biodiversidad: protocolos y aplicaciones. Bogotá: Instituto de Investigación de Recursos Biológicos Alexander von Humboldt. 236 p. 
Schneider, C., Rasband, W., \& Eliceiri, K. (2012). ImageJ. "NIH Image to ImageJ: 25 years of image analysis". Nature methods, 9(7), 671-675. DOI: https:// doi.org/10.1038/nmeth.2089

Solorza, J. (2016). Investigación en áreas piloto de restauración ecológica ("Apires"). Scientific journal, Jardín Botánico de Bogotá José Celestino Mutis. Bio-Síntesis, 1(1), 5.

Squeo, F., Olivares, N., Olivares, S., Pollastri, A., Aguirre, E., Aravena, R., Jorquera, C., \& Ehleringer, J. (1999). Grupos funcionales en arbustos desérticos del norte de Chile, definidos sobre la base de las fuentes de agua utilizadas. Gayana Botanica, 56(1), 1-15.

The Angiosperm Phylogeny Group. (2009), An update of the Angiosperm Phylogeny Group classification for the orders and families of flowering plants: APG III. Botanical Journal of the Linnean Society, 161, 105-121. DOI: https://doi. org/10.1111/j.1095-8339.2009.00996.x

Violle, C., Navas, M., Vile, D., Kazakou, E., Fortunel, C., Hummel, I., \& Garnier, E. (2007). Let the concept of trait be functional. Oikos, 116, 882-892. DOI: https://doi.org/10.1111/j.0030-1299.2007.15559.x
Warnock, R., Valenzuela, J., Trujillo, A., Madriz, P., \& Gutiérrez, M. (2006). Área foliar, componentes del área foliar y rendimiento de seis genotipos de Caraota. Agronomía Tropical, 56(1), 21-42.

Webb, C., Ackerly, D., \& Kembel, S. (2008). Phylocom: software for the analysis of phylogenetic community structure and trait evolution. Bioinformatics, 24, 2098-2100. DOI: https://doi.org/10.1093/ bioinformatics/btn358

Wright, I., Reich, P., Westoby, M., Ackerly, D., Baruch, Z., Bongers, F., \& Cavender-Bares, J. (2004). The worldwide leaf economics spectrum. Nature, 428(6985), 821-827. DOI: https://doi.org/10.1038/ nature02403

Wilson, P., Thompson, K., \& Hodgson, J. (1999). Specific leaf area and leaf dry matter content as alternative predictors of plant strategies. New Phytologist, 143(1), 155-162. DOI: https://doi. org/10.1046/j.1469-8137.1999.00427.x

Wortley, L., Hero, J., \& Howes, M. (2013). Evaluating ecological restoration success: A review of the literature. Restoration Ecology, 21, 537-543. DOI: https://doi.org/10.1111/rec.12028 